\title{
Guest Editorial: Selected Papers from ISAAC 2017
}

\section{Yoshio Okamoto ${ }^{1}$}

Published online: 10 January 2020

๑) Springer Science+Business Media, LLC, part of Springer Nature 2020

This issue contains selected papers from the 28th International Symposium on Algorithms and Computation (ISAAC 2017) held in Phuket, Thailand, on December 9-12, 2017. The ISAAC 2017 Program Committee, co-chaired by Takeshi Tokuyama and myself, accepted 65 papers among 190 high-quality submissions ${ }^{1}$ (with three submissions withdrawn), after a rigorous review process. Among those accepted papers, the following ten papers have been selected in this special issue. They went through the standard refereeing process of Algorithmica before being accepted for publication.

- Tilt Assembly: Algorithms for Micro-Factories that Build Objects with Uniform External Forces by Aaron T. Becker, Sándor P. Fekete, Phillip Keldenich, Dominik Krupke, Christian Rieck, Christian Scheffer, and Arne Schmidt

- A Simple Greedy Algorithm for Dynamic Graph Orientation by Edvin Berglin and Gerth Stølting Brodal

- Crossing Number for Graphs with Bounded Pathwidth by Therese Biedl, Markus Chimani, Martin Derka, and Petra Mutzel

- An Improved Algorithm for Computing All the Best Swap Edges of a Tree Spanner by Davide Bilò, Feliciano Colella, Luciano Gualà, Stefano Leucci, and Guido Proietti

- On the Convergence Time of a Natural Dynamics for Linear Programming by Vincenzo Bonifaci

- Study of a combinatorial game in graphs through Linear Programming by Nathann Cohen, Fionn Mc Inerney, Nicolas Nisse, and Stéphane Pérennes

- On Using Toeplitz and Circulant Matrices for Johnson-Lindenstrauss Transforms by Casper Benjamin Freksen and Kasper Green Larsen

- Maximum Induced Matching Algorithms via Vertex Ordering Characterizations by Michel Habib and Lalla Mouatadid

\footnotetext{
1 The frontmatter of the ISAAC 2017 proceedings [1] contains "170" as the number of submissions, but this number is incorrect. The correct number of submissions is 190. I apologize for the confusion.

Yoshio Okamoto

okamotoy@uec.ac.jp

1 The University of Electro-Communications, Chofugaoka 1-5-1, Chofu, Tokyo 182-8585, Japan
} 
- Fast Compressed Self-Indexes with Deterministic Linear-Time Construction by J. Ian Munro, Gonzalo Navarro, and Yakov Nekrich

- Envy-free Matchings with Lower Quotas by Yu Yokoi

We wish to thank the authors for submitting their papers to the special issue. We also thank the referees for their thorough reviews and valuable comments that helped to improve many aspects of the papers published in this issue.

\section{References}

1. Okamoto, Y., Tokuyama, T.: Front Matter, Table of Contents, Preface, External Reviewers. In: Proceedings of the 28th International Symposium on Algorithms and Computation (ISAAC 2017), Leibniz International Proceedings in Informatics (LIPIcs), vol. 92, pp. 0:i-0:xvi (2017)

Publisher's Note Springer Nature remains neutral with regard to jurisdictional claims in published maps and institutional affiliations. 